\title{
Combination of Root Surface Modification with BMP-2 and Collagen Hydrogel Scaffold Implantation for Periodontal Healing in Beagle Dogs
}

\author{
Akihito Kato ${ }^{1}$, Hirofumi Miyaji ${ }^{1, *}$, Ryosuke Ishizuka ${ }^{1}$, Keisuke Tokunaga ${ }^{1}$, Kana Inoue ${ }^{1}$, \\ Yuta Kosen ${ }^{1}$, Hiroyuki Yokoyama ${ }^{1}$, Tsutomu Sugaya ${ }^{1}$, Saori Tanaka ${ }^{1}$, Ryuji Sakagami ${ }^{2}$ and \\ Masamitsu Kawanami ${ }^{1}$
}

${ }^{I}$ Department of Periodontology and Endodontology, Hokkaido University Graduate School of Dental Medicine,
Sapporo, Japan; ${ }^{2}$ Section of Periodontology, Department of Odontology, Fukuoka Dental College, Fukuoka, Japan

\begin{abstract}
Objective: Biomodification of the root surface plays a major role in periodontal wound healing. Root surface modification with bone morphogenetic protein (BMP) stimulates bone and cementum-like tissue formation; however, severe ankylosis is simultaneously observed. Bio-safe collagen hydrogel scaffolds may therefore be useful for supplying periodontal ligament cells and preventing ankylosis. We examined the effects of BMP modification in conjunction with collagen hydrogel scaffold implantation on periodontal wound healing in dogs. Material and Methods: The collagen hydrogel scaffold was composed of type I collagen sponge and collagen hydrogel. One-wall infrabony defects $(5 \mathrm{~mm}$ in depth, $3 \mathrm{~mm}$ in width) were surgically created in six beagle dogs. In the BMP/Col group, BMP-2 was applied to the root surface (loading dose; $1 \mu \mathrm{g} / \mu \mathrm{l}$ ), and the defects were filled with collagen hydrogel scaffold. In the BMP or Col group, BMP-2 coating or scaffold implantation was performed. Histometric parameters were evaluated at 4 weeks after surgery. Results: Single use of BMP stimulated formation of alveolar bone and ankylosis. In contrast, the BMP/Col group frequently enhanced reconstruction of periodontal attachment including cementum-like tissue, periodontal ligament and alveolar bone. The amount of new periodontal ligament in the BMP/Col group was significantly greater when compared to all other groups. In addition, ankylosis was rarely observed in the BMP/Col group. Conclusion: The combination method using root surface modification with BMP and collagen hydrogel scaffold implantation facilitated the reestablishment of periodontal attachment. BMP-related ankylosis was suppressed by implantation of collagen hydrogel.
\end{abstract}

Keywords: Ankylosis, bone morphogenetic protein-2, collagen hydrogel, dog, one-wall infrabony defects, periodontal attachment, periodontal wound healing, regenerative scaffold.

\section{INTRODUCTION}

In periodontal regenerative therapy, stable periodontal attachment, including cementum and Sharpey's fibers, should be a guide for reforming the instrumented root surface. However, it is difficult to achieve these objectives due to rapid junctional epithelium downgrowth, which prevents the formation of periodontal attachment [1]. Even if epithelial tissue does not invade the root surface after healing, many cases show gingival tissue adaptation without periodontal attachment apparatus [2]. Therefore, compatibility between the root surface and regenerated periodontal tissue is required for a predictable regenerative procedure.

Bio-modification of the root dentin surface plays a major role in periodontal healing. Many investigators have confirmed that agents for dentin demineralization remove the surface smear layer, open dentin tubules and expose organic elements such as the collagen matrix, thus increasing total surface area [3, 4]. Various modifications provide a more biocompatible dentin surface; protein absorption, cell

\footnotetext{
*Address correspondence to this author at the Department of Periodontology and Endodontology, Hokkaido University Graduate School of Dental Medicine, N13 W7 Kita-ku, Sapporo 060-8586, Japan; Tel: +81 11706 4266; Fax: +81 11706 4334;

E-mail: miyaji@den.hokudai.ac.jp
}

migration and attachment and fiber development [5-8]. Furthermore, demineralized dentin is a suitable surface for retention of growth and differentiation factors [6, 7]. Bone morphogenetic proteins (BMPs), known to be biological differentiation factors, have the ability to transform pluripotent stem cells into osteoprogenitor cells [9] and to promote ectopic osteogenesis in the body [10,11]. Zaman et al. reported that BMP-applied dentin stimulated the osteogenic activity of attached human periodontal ligament cells [12]. Miyaji et al. presented an in vivo study in which cementumlike tissue was directly formed on the BMP-applied dentin surface in gingival connective tissue $[13,14]$. In addition, root surface modification with BMP markedly prevented epithelial downgrowth in experimental periodontal defects in dogs [15]. Therefore, root biomodification using BMPs increases bioactivity and should promote the re-establishment of stable periodontal attachment including cementum and alveolar bone.

However, Miyaji et al. also demonstrated that root surface modification with BMP frequently causes severe ankylosis and there is little evidence of periodontal ligament formation [16]. Ankylosis refers to aberrant healing following periodontal therapy, i.e., regenerated bone binds directly to the instrumented root surface. Ankylosis is frequently caused by highly effective medicines and/or biomaterials for bone 
tissue engineering [16], and many investigators have demonstrated that reformation of the periodontal ligament synchronously reduces the occurrence of ankylosis [16, 17]. BMPs show high proliferative activity on osteoblasts, but low activity on human periodontal ligament cells [12]. In addition, BMP-2 exhibits up-regulation of alkaline phosphatase activity and mineralization of periodontal ligament cells [18]. Therefore, released BMPs from the root surface may trigger severe ankylosis. Accordingly, a cell source for periodontal ligaments is required to prevent ankylosis in the root biomodification procedure using BMPs.

Recently, tissue engineering approaches have been introduced and expected for clinical applications. Histologic evaluation of periodontal supporting structures was performed following the application of periodontal cell sheets [19] and stem cells [20]. These methods are very predictable, but very complex and expensive. On the other hand, the scaffold is able to provide an optimal environment for cell migration and proliferation into regenerative spaces [21]. Hydrated polymers, such as hydrogel, are an effective scaffold material consisting of synthetic and/or natural copolymers [22]. Previous reports have revealed that activity of periodontal ligament cells is stimulated by Type I collagen application [23]. Therefore, collagen hydrogel scaffolds may be useful for supplying periodontal ligament cells. It is an inexpensive material, in contrast to cell transplantation, and may therefore be widely used for clinical trials. In vitro and in vivo studies have demonstrated ingrowth of fibroblasts, including periodontal ligament cells and vascular endothelial cells into hydrated collagen gels $[24,25]$. In addition, biosafety profiles for collagen hydrogel scaffolds have been reported; they exhibit high degradability, no toxicity and no chronic inflammatory response [26, 27]. In dog periodontal healing, applied collagen hydrogel enhanced the growth of cell-rich connective tissue continuous with the pre-existing periodontal ligament along with the root surface [26]. The collagen hydrogel scaffold also enhanced the formation of new cementum and periodontal ligament, as well as alveolar bone; however, ankylosis was not detected [27, 28]. Collagen hydrogel scaffolds may selectively induce proliferation of periodontal ligament cells for regenerating the attachment apparatus. Thus, we hypothesized that collagen hydrogels provide numerous periodontal ligament cells around the instrumented root, form stable periodontal attachments and inhibit the ankylosis associated with BMP application. In large periodontal defects, however, a combined design for periodontal healing has not yet been investigated. Accordingly, the aim of this study was to histologically evaluate whether a combination method using root surface modification with BMP-2 and collagen hydrogel scaffold facilitates periodontal wound healing in one-wall infrabony defects in beagle dogs.

\section{MATERIALS AND METHODS}

\section{Preparation and Morphological Analysis of Collagen Hydrogel/Sponge Scaffold}

Collagen hydrogel was prepared from atelocollagen derived from calf skin dermis (Koken, Tokyo, Japan). Atelocollagen solution was stirred with $1 \mathrm{M} \mathrm{HCl}$ and stored at $10^{\circ} \mathrm{C}$ for 3 days. Subsequently, $1 \mathrm{mM} \mathrm{L}(+)$-ascorbic acid and $0.1 \mathrm{mM} \mathrm{CuCl}_{2}$ were added to this solution, which was then adjusted to a final concentration of $1.5 \%$ to prepare collagen hydrogel (Fig. 1A).

Collagen sponge was provided by Olympus Terumo Biomaterials (Tokyo, Japan). Collagen sponge was prepared as follows. Atelocollagen in a dilute $\mathrm{HCl}$ solution was neutralized by adding concentrated phosphate buffer in $\mathrm{NaCl}$ to a final concentration of $0.1 \%$ collagen, $30 \mathrm{mM} \mathrm{Na}_{2} \mathrm{HPO}_{4}$ and $0.1 \mathrm{M} \mathrm{NaCl}$. This collagen solution was incubated at $37^{\circ} \mathrm{C}$ for 4 hours. The resulting fibrous precipitate was referred to as fibrillar collagen (FC). Heat-denatured collagen (HAC) was prepared from atelocollagen in a dilute $\mathrm{HCl}$ solution by heating at $60^{\circ} \mathrm{C}$ for 30 minutes. A composite of FC and HAC was prepared by mixing the two at a ratio of $9: 1(\mathrm{w} / \mathrm{w})$, respectively. This composite material was adjusted to a final concentration of $4 \%$ and made into the form of a sponge by lyophilization at $-30^{\circ} \mathrm{C}$. This sponge was dehydrothermally cross-linked at $110^{\circ} \mathrm{C}$ for 2 hours and used as a collagen sponge. In this study, 36 pieces of collagen sponge $(5 \times 3 \times 3$ $\mathrm{mm}$ ) were used (Fig. 1B).

Consequently, collagen hydrogel $(100 \mu \mathrm{l})$ was injected into the collagen sponge block under vacuum (Fig. 1C). For morphological analysis, collagen hydrogel scaffold was fixed in $10 \%$ buffered formalin and embedded in paraffin. Sections $(6 \mu \mathrm{m})$ were prepared, stained with hematoxylin and eosin (HE) and examined by light microscopy.

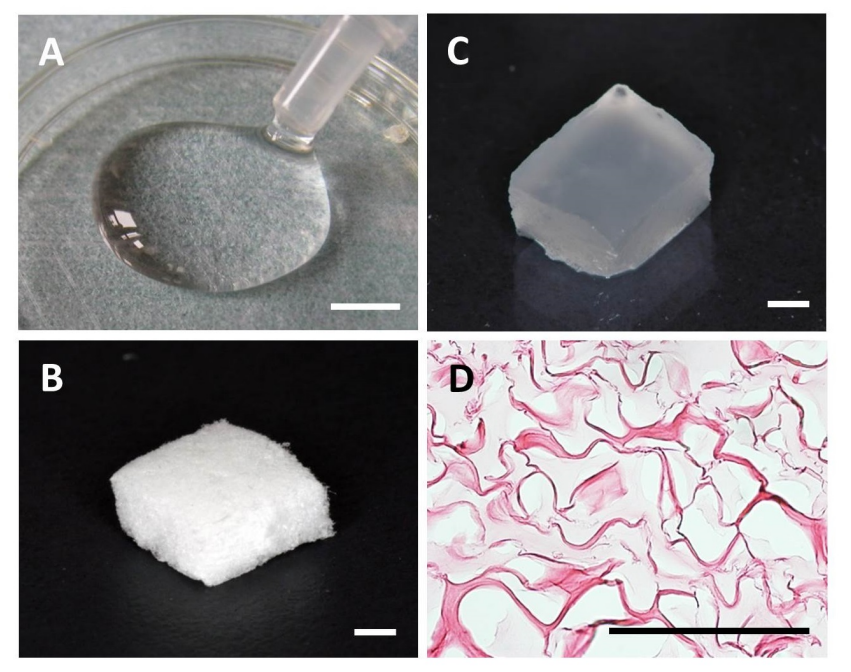

Fig. (1). (A) Collagen hydrogel. (B) Collagen sponge. (C) Collagen hydrogel scaffold. (D) Hematoxylin and eosin staining of collagen hydrogel scaffold. Scale bars: $(\mathbf{A})=5 \mathrm{~mm} ;(\mathbf{B}$ and $\mathbf{C})=1 \mathrm{~mm}$; (D) $=100 \mu \mathrm{m}$.

\section{BMP-2 Construct}

Recombinant human BMP-2 (98\% purity) donated by Astellas Pharma (Tokyo, Japan) was used in this study. BMP-2 was diluted with phosphate-buffered saline (PBS; 5 $\mathrm{mM}, \mathrm{pH} 7.2$ ) to produce stock solutions of $1.0 \mu \mathrm{g} / \mu \mathrm{l}$.

\section{Animals}

Six healthy female beagle dogs (age, 12-16 months; weight, approximately $10 \mathrm{~kg}$ ), were used in this experiment. Experimental protocols conformed to the institutional animal care and use regulations of Hokkaido University (Animal 
Research Committee of Hokkaido University, Approval No. 08-0255). Surgical procedures were performed under general anesthesia with medetomidine hydrochloride $(0.1 \mathrm{ml} / \mathrm{kg}$; Domitor, Nippon Zenyaku Kogyo, Koriyama, Japan) and butorphanol tartrate $(0.1 \mathrm{ml} / \mathrm{kg}$, Vetorphale, Meiji Seika, Tokyo, Japan), and under local anesthesia with lidocaine hydrochloride (2\% with 1:80,000 epinephrine; Xylocaine, Dentsply-Sankin, Tokyo, Japan).

\section{Surgical Procedure}

Mandibular first and third premolars were extracted before experimental surgery, and extraction sites were allowed to heal for 8 weeks. Following reflection of the buccal and lingual muco-gingival flaps with partial thickness, periosteum was removed from alveolar bone. Thirty-six onewall infrabony defects (depth: $5 \mathrm{~mm}$; width: $3 \mathrm{~mm}$ ) were surgically created in the mesial and distal aspects of the mandibular second premolars, and the mesial aspect of the mandibular fourth premolars (Fig. 2A). The root surface facing the defect was planed to remove cementum. Reference notches indicating the cemento-enamel junction and bottom of the defect were prepared on root surfaces. The denuded root surface was demineralized with 24\% EDTA ( $\mathrm{pH} 7.0$ ) for 3 minutes and washed with saline. Subsequently, defects were randomly assigned to four groups. In the $\mathrm{BMP} / \mathrm{Col}$ group, $25 \mu \mathrm{l}$ of recombinant human BMP-2 solution (loading dose; $1 \mu \mathrm{g} / \mu \mathrm{l}$ ) was applied to the root surface (Fig. 2B), and the defect was filled with collagen hydrogel scaffold (Fig. 2C). In the BMP or Col group, BMP-2 coating was performed or the defect was filled with collagen hydrogel scaffold. In the control, neither conditioning nor implantation was performed. The flap was repositioned and securely sutured (Surgilon; Tyco Healthcare Japan, Tokyo, Japan; Fig. 2D). Animals received ampicillin sodium $(300 \mathrm{mg} / \mathrm{kg}$; Viccillin, Meiji Seika, Tokyo, Japan) daily for 3 days, and a plaque control regimen with $0.5 \%$ chlorhexidine twice weekly for the entire duration of the experiment.
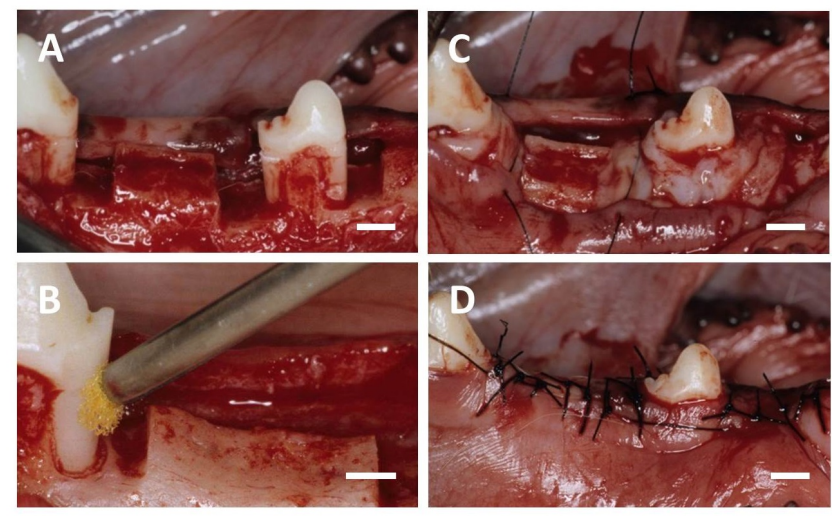

Fig. (2). (A) After the flap was elevated, one-wall infrabony defects were surgically created. (B) BMP-2 solution was applied to the root surface. (C) The defect was filled with collagen hydrogel scaffold. (D) The flap was repositioned and securely sutured. Scale bars: (A, B, $\mathbf{C}$ and $\mathbf{D})=3 \mathrm{~mm}$.

\section{Histological Procedure}

Animals were euthanized using an overdose of sodium pentobarbital $(0.5 \mathrm{ml} / \mathrm{kg}$, Somnopentyl, Kyoritsu, Tokyo,
Japan) following general anesthesia with medetomidine hydrochloride and butorphanol tartrate. Specimens were collected from the wound at 4 weeks post-surgery. Tissue blocks, including teeth, bone and soft tissue, were fixed in $10 \%$ buffered formalin, decalcified in $10 \%$ formic-citric acid, and embedded along the mesial-distal plane in paraffin wax. Sections (6 $\mu \mathrm{m}$ thick) were serially prepared and stained with hematoxylin-eosin (HE) and Masson's trichrome.

\section{Histomorphometric Analysis}

Three stained sections were taken; one was approximately from the center of the root, and the other two were $180 \mu \mathrm{m}$ from either side of the center. The following nine histomorphometric measurements (Fig. 3) were performed for each section using a software package (Image J 1.41; National Institutes of Health, Bethesda, MD):

1. Defect height: distance between the apical notch and the cemento-enamel junction.

2. Defect width: length of the bottom of the osseous defect.

3. New cementum: distance between the apical notch and the coronal extension of the newly formed cementumlike tissue on the root surface.

4. New periodontal ligament: length of functional fibrous tissue between the newly formed cementum-like tissue and alveolar bone.

5. New bone height: distance between apical notch and coronal extension of newly formed alveolar bone along the root surface.

6. New bone area: newly formed alveolar bone in defect area.

7. Ankylosis: length of ankylotic union of newly formed alveolar bone and root surface.

8. Gingival connective tissue: distance between apical extension of junctional epithelium and coronal extension of alveolar bone or cementum.

9. Junctional epithelium: distance between cemento-enamel junction and apical extension of junctional epithelium.

10. Gingival recession: distance between cemento-enamel junction and gingival margin.

We also counted the frequency of ankylosis, epithelium downgrowth and gingival recession in each group.

\section{Statistical Analysis}

The means and standard deviations of each parameter were calculated for four groups. Differences between the groups were analyzed using Scheffé test. P-values $<0.05$ were considered to be statistically significant. All statistical procedures were performed using a software package (DR.SPSS 11.0; SPSS Japan, Tokyo, Japan).

\section{RESULTS}

\section{Morphology of Collagen Hydrogel Scaffold}

On microscopy, collagen hydrogel was present as an amorphous structure and had fully penetrated into the collagen sponge (Fig. 1D). 


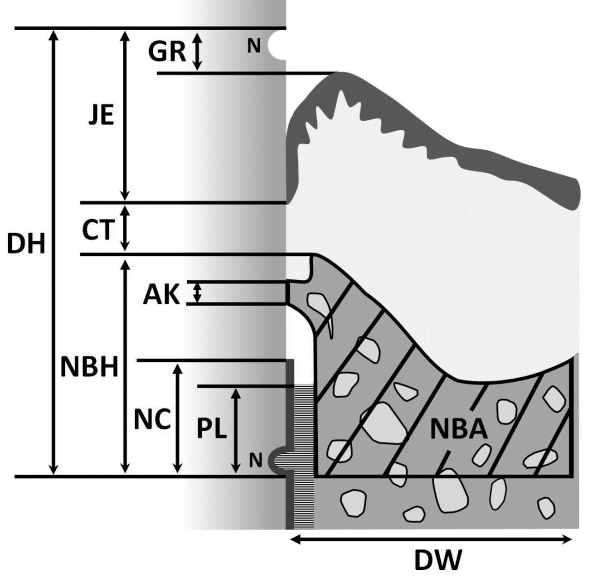

Fig. (3). Schematic drawing of histomorphometric analysis of periodontal wound healing. (DH, defect height; DW, defect width; $\mathrm{NC}$, new cementum; PL, new periodontal ligament; $\mathrm{NBH}$, new bone height; NBA, new bone area; AK, ankylosis; $\mathrm{CT}$, gingival connective tissue; JE, junctional epithelium; GR, gingival recession; N, notches).

\section{Histological Observations}

Due to pulp tissue exposure during the creation of bone defects in the surgical procedure, two specimens were excluded from this study. Otherwise, postoperative healing was uneventful in all dogs.

\section{BMP Group}

BMP application enhanced periodontal tissue reconstruction (Fig. 4A). New cementum-like tissue was frequently formed on the BMP-applied root surface. Cementum-like tissue was relatively thick with a layered structure, i.e., cement line, including cementocyte-like cells, showing the appearance of cellular intrinsic-fiber cementum (Fig. 4B). Cementum-like tissue was covered with cementoblastic cells; however, Sharpey's fibers associated with periodontal ligament formation were not observed. In addition, cells and extracellular matrix were poorly distributed in the interface between new alveolar bone and cementum-like tissue. Masson's staining clearly indicated the lack of fibrous tissue along the root surface (Fig. 4C). Although alveolar bone formation occurred parallel to the root surface, we frequently observed ankylotic union (Fig. 4D). Newly formed woven bone morphologically included osteoblasts, osteocytes and bone marrow related to native bone. Epithelial downgrowth was suppressed in the coronal portion of the root surface. BMP group specimens did not receive regenerative scaffold application, but severe gingival recession was not observed.

\section{Col Group}

Following collagen hydrogel application, periodontal healing was observed; however, there were some differences between the Col and BMP groups (Fig. 5A). We frequently detected acellular cementum-like tissue continuous with the original cementum on the instrumented root surface (Fig. 5C). In contrast to the BMP group, cementum-like tissue appeared as a thin layer and with an unclear cement line. Periodontal ligaments were reformed and well-stained as the collagen-rich layer (Fig. 5D). Sharpey's fibers inserting into both the new cementum-like tissue and alveolar bone were observed, indicating that functionally oriented periodontal ligament tissue was reestablished. Ankylosis was rarely observed when compared to the BMP group. In the coronal portion, gingival connective tissue attached to the root surface. Some residual collagen hydrogel scaffold was observed around the new alveolar bone, fractionated by newly formed connective tissue (Fig. 5B). As a result, fibroblastic and osteoblastic cells had proliferated on the inner side of the collagen hydrogel material. Few inflammatory cells were also seen around the residual collagen scaffold. Gingival recession was mild, similar to that of the BMP group.

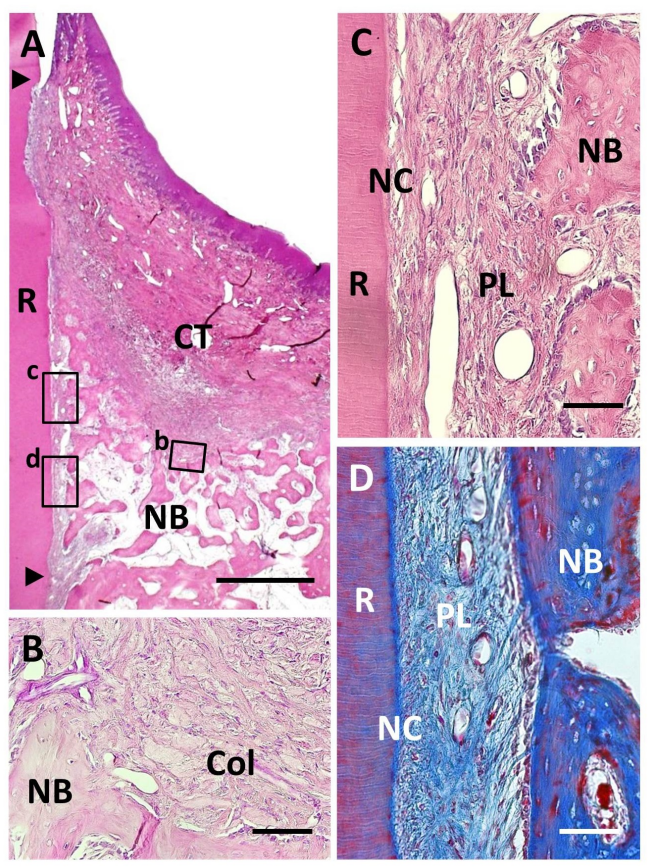

Fig. (4). Histological findings in BMP group. (A) Alveolar bone formation occurred along the root surface. Coronal and apical notches are indicated by arrowheads. (B) Higher magnification of framed area (b) in (A). New cellular cementum was detected on the BMP-treated root surface. (C) Higher magnification of framed area (c) in (A). Little periodontal ligament formation was observed between new bone and cementum. (D) Higher magnification of framed area (d) in (A). Ankylosis was frequently noted. R, root; $\mathrm{CT}$, gingival connective tissue; NB, new bone; NC, new cementum; AK, ankylosis. Staining: hematoxylin and eosin (A, B, D) and Masson's trichrome $(\mathbf{C})$. Scale bars: $(\mathbf{A})=1 \mathrm{~mm} ;(\mathbf{B})=25 \mu \mathrm{m} ;(\mathbf{C}$ and D) $=50 \mu \mathrm{m}$.

\section{BMP/Col Group}

We found that root surface modification with BMP in combination with collagen hydrogel scaffold implantation facilitated periodontal wound healing (Fig. 6A). Considerable new alveolar bone occupied the periodontal defect space. New bone was composed of narrow trabeculae including osteoblasts and osteocytes. Thick, cellular cementumlike tissue was frequently evident on the BMP-applied root surface, even in the coronal portion (Fig. 6B). Cementumlike tissue was lined with cementoblastic cells and showed Sharpey's fiber insertion. Despite BMP loading, fiber-rich periodontal ligaments were reestablished, in contrast to specimens in the BMP group (Fig. 6C and D). Although 


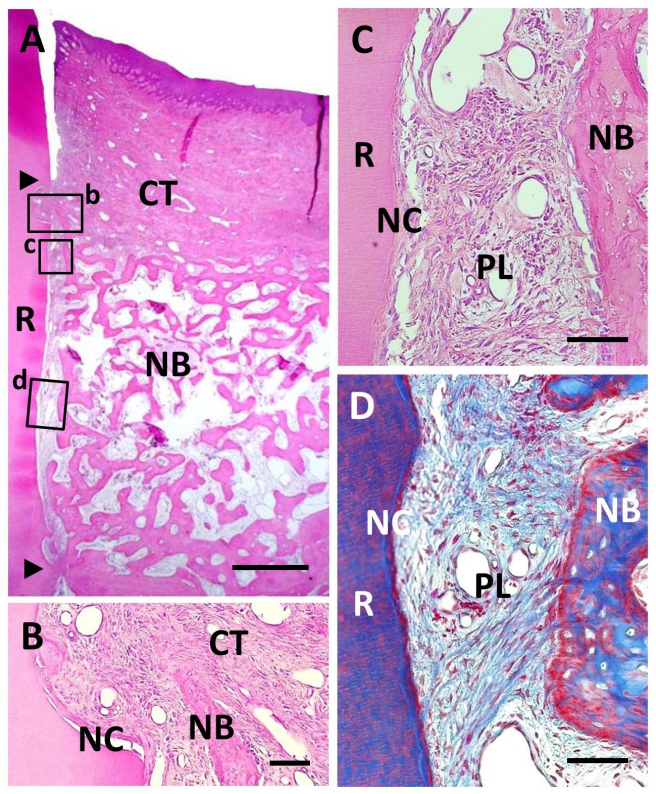

Fig. (5). Histological findings in Col group. (A) Formation of new bone was observed in the defect. Coronal and apical notches are indicated by arrowheads. (B) Higher magnification of framed area (b) in (A). Cell ingrowth was observed in the residual collagen hydrogel scaffold. (C) Higher magnification of the framed area (c) in (A). Thin layer of cementum-like tissue was observed on the root dentin surface. (D) Higher magnification of the framed area (d) in (A). Periodontal ligament tissue with functionally oriented fibers was reestablished between the new bone and new cementum-like tissue. $\mathrm{R}$, root; $\mathrm{CT}$, gingival connective tissue; $\mathrm{NB}$, new bone; $\mathrm{NC}$, new cementum; Col, Collagen hydrogel scaffold; PL, periodontal ligament. Staining: hematoxylin and eosin $(\mathbf{A}, \mathbf{B}, \mathbf{C})$ and Masson's trichrome (D). Scale bars: $(\mathbf{A})=1 \mathrm{~mm} ;(\mathbf{B}, \mathbf{C}$ and $\mathbf{D})=50 \mu \mathrm{m}$.

marked alveolar bone formation was observed along the BMP-modified root surface, ankylosis was reduced when compared with the BMP group. Residual collagen hydrogel scaffolds were rarely seen in the defect, suggesting that the scaffold exhibited high degradability. Regulation of epithelial downgrowth and gingival recession was favorable.

\section{Control Group}

Bioactivity for reconstruction of periodontal attachment was not stimulated in control specimens. Newly formed connective tissues composed of infiltrating fibroblastic cells and blood vessels filled the defect in most areas (Fig. 7A). Formation of alveolar bone, cementum and periodontal ligament were limited only in the apical portion of the defect (Fig. 7B). No ankylosis or root resorption was observed. In the coronal portion, there was no evidence of cementum or periodontal ligament formation, and gingival tissue adhesion was seen. Epithelial downgrowth and gingival recession were frequently observed (Fig. 7C).

\section{Histomorphometric Analysis}

The degree of periodontal healing in one-wall infrabony defects is presented in Table 1. There were no significant differences among mean defect height and width in each group. Combination therapy using BMP and collagen hydro-

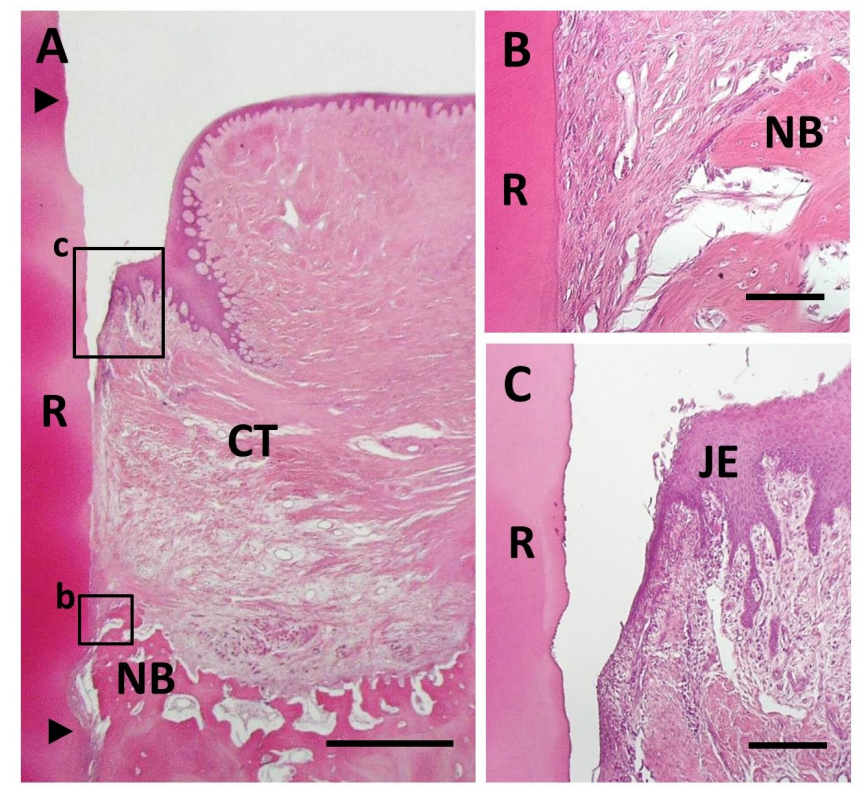

Fig. (6). Histological findings in $\mathrm{BMP} / \mathrm{Col}$ group. (A) Extensive alveolar bone regeneration was observed in the defect area. Coronal and apical notches are indicated by arrowheads. (B) Higher magnification of framed area (b) in (A). Cementum-like tissue was frequently evident on the BMP-applied root surface in coronal portion. (C) Higher magnification of framed area (c) in (A). Fiber-rich periodontal ligament tissue was re-established between cementum-like tissue and alveolar bone. (D) Higher magnification of framed area (d) in (A). Sharpey's fibers inserting into both new alveolar bone and cementum-like tissue were seen. $\mathrm{R}$, root; $\mathrm{CT}$, gingival connective tissue; NB, new bone; NC, new cementum; PL, periodontal ligament. Staining: hematoxylin and eosin $(\mathbf{A}, \mathbf{B}, \mathbf{C})$ and Masson's trichrome (D). Scale bars: $(\mathbf{A})=1 \mathrm{~mm} ;(\mathbf{B}, \mathbf{C}$ and $\mathbf{D})=50 \mu \mathrm{m}$.

gel scaffold facilitated periodontal healing, as compared to the use of each alone. In specimens in the BMP/Col group, cementum-like tissue and periodontal ligaments associated with stable periodontal attachment showed significant reconstruction when compared to the control and BMP groups. In the Col group, periodontal attachment was stimulated; however, its levels in the $\mathrm{BMP} / \mathrm{Col}$ group were approximately 1.5 -fold greater than in the Col group. In the BMP group, significantly more cementum-like tissue was observed when compared to the control group, but periodontal ligament formation was not accelerated.

Bone conducting parameters showed that BMP and collagen hydrogel application greatly promoted formation of alveolar bone. The bone forming effects in the BMP/Col group were the most active of all groups. We also found greater new bone height and area in the BMP and Col groups as compared to controls; however, the BMP group frequently showed ankylosis. The frequency of abnormal healing, including ankylosis, epithelial downgrowth and gingival recession, is shown in Table 2. Ankylosis was evident in six defect sites in the BMP group, suggesting that periodontal healing was unpredictable in BMP coating therapy. Specimens receiving collagen hydrogel scaffold demonstrated a little bone adhesion, regardless of root surface modification by BMP. Accordingly, hydrated collagen application clearly suppressed BMP-related ankylosis. 


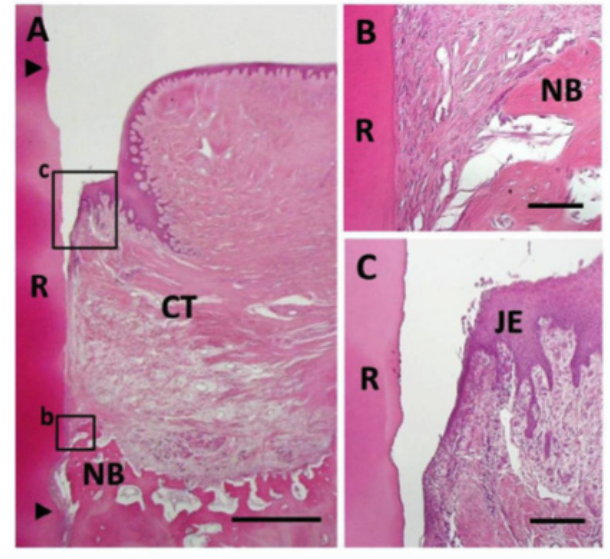

Fig. (7). Histological findings in control group. (A) Newly formed connective tissue filled most defect areas. Coronal and apical notches are indicated by arrowheads. (B) Higher magnification of framed area (b) in (A). Bone and cementum formation was limited in the apical portion of the defect. (C) Higher magnification of framed area (c) in (A). Downgrowth of junctional epithelium was frequently seen. R, root; $\mathrm{CT}$, gingival connective tissue; $\mathrm{NB}$, new bone; JE, Junctional epithelium. Staining: hematoxylin and eosin $(\mathbf{A}, \mathbf{B}, \mathbf{C})$. Scale bars: $(\mathbf{A})=1 \mathrm{~mm} ;(\mathbf{B})=50 \mu \mathrm{m} ;(\mathbf{C})=100 \mu \mathrm{m}$.

There were no significant differences in junctional epithelium and gingival recession among the groups. However, the BMP-treated group tended to show decreased epithelial downgrowth. In addition, experimental groups showed a low frequency of gingival recession.

\section{DISCUSSION}

The present study focused on the effects of BMPbiomodification in conjunction with collagen hydrogel scaffold implantation for developing periodontal attachment. We demonstrated that use of BMP modification led to ankylosis. Currently, BMP therapy is performed in combination with the application of various biological scaffolds [29, 30]. A scaffold loaded with BMP directly filled the periodontal osseous defect $[31,32]$, allowing osteoconductive cells to accumulate on the root surface. BMP osteogenic properties, such as osteoblast differentiation, proliferation and migration, have been studied extensively [9, 10]. Furthermore, BMPs suppressed proliferative activity of human periodontal ligament cells [12]. Sigurdsson et al. assessed periodontal healing after implantation of several BMP-loaded scaffolds, e.g., bone matrix, collagen sponge, poly(lactic-co-glycolic acid) and poly lactic acid. They found that induction of ankylosis applied to all scaffold cases [33]. Therefore, the manner of BMP application in periodontal disease should aim to improve BMP-related ankylosis.

We assumed that the key to regulation of ankylosis and predictable periodontal attachment is localizing BMP and its cell source in the defect. Several studies have designed methods for BMP localization, i.e., instrumented root surfaces are fully covered with biomaterials having no BMP and defects are filled with BMP-loaded materials, in an effort to separate BMP effects from the root and to prevent populating of osteoblastic cells around the root surface [17]. In contrast, this examination applied BMP to the root surface and not to the periodontal defect in anticipation of the proliferation of periodontal ligament cells throughout the defect. Subsequently, proliferating periodontal cells should be attached to the BMP-modified root surface and then form hard tissues on the root. The results of this study showed that periodontal ligaments with cementum-like tissue are favorably re-established on BMP-applied root surfaces and that ankylosis is rarely observed. According to these results, we believe that BMP modification with a sufficient population of periodontal cells specifically inhibited the occurrence of ankylosis.

In the group receiving collagen hydrogel scaffold, formation of periodontal ligaments was frequently observed, regardless of BMP application. Periodontal fibroblastic cells grow well in hydrated collagen gel [34]. Kosen et al. reported that periodontal ligament, including Sharpey's fibers,

Table 1. Histomorphometric analysis (means \pm SD).

\begin{tabular}{|c|c|c|c|c|}
\hline & $\begin{array}{l}\text { Control group } \\
(n=9)\end{array}$ & $\begin{array}{l}\text { BMP group } \\
(n=9)\end{array}$ & $\begin{array}{l}\text { Col group } \\
\quad(n=8)\end{array}$ & $\begin{array}{c}\text { BMP/Col group } \\
(n=8)\end{array}$ \\
\hline Defect height (mm) & $4.73 \pm 0.21$ & $4.55 \pm 0.24$ & $4.91 \pm 0.36$ & $4.79 \pm 0.40$ \\
\hline Defect width (mm) & $2.97 \pm 0.17$ & $3.04 \pm 0.13$ & $3.19 \pm 0.17$ & $3.07 \pm 0.19$ \\
\hline New cementum (mm) & $0.34 \pm 0.26$ & $1.47 \pm 0.61^{*}$ & $1.84 \pm 0.79^{*}$ & $2.62 \pm 0.70 * \dagger$ \\
\hline New periodontal ligament $(\mathrm{mm})$ & $0.32 \pm 0.25$ & $0.86 \pm 0.34$ & $1.34 \pm 0.62 *$ & $2.21 \pm 0.77 *^{\dagger \S}$ \\
\hline New bone height (mm) & $0.81 \pm 0.30$ & $2.82 \pm 0.69^{*}$ & $1.88 \pm 0.56^{*}$ & $3.06 \pm 1.08 * \S$ \\
\hline New bone area $\left(\mathrm{mm}^{2}\right)$ & $1.64 \pm 0.65$ & $4.16 \pm 0.85^{*}$ & $4.56 \pm 1.07 *$ & $7.64 \pm 3.12 * \dagger \S$ \\
\hline Ankylosis (mm) & $0.00 \pm 0.00$ & $0.53 \pm 0.83$ & $0.03 \pm 0.08$ & $0.04 \pm 0.11$ \\
\hline Gingival connective tissue (mm) & $2.91 \pm 0.93$ & $1.53 \pm 0.50^{*}$ & $1.58 \pm 1.04^{*}$ & $1.33 \pm 0.69^{*}$ \\
\hline Junctional epithelium (mm) & $0.94 \pm 0.90$ & $0.20 \pm 0.30$ & $1.04 \pm 0.95$ & $0.40 \pm 0.50$ \\
\hline Gingival recession (mm) & $0.34 \pm 0.49$ & $0.02 \pm 0.07$ & $0.13 \pm 0.35$ & $0.00 \pm 0.00$ \\
\hline
\end{tabular}

* Statistical difference vs. control group $(\mathrm{p}<0.05)$.

${ }^{\dagger}$ Statistical difference vs. BMP group $(\mathrm{p}<0.05)$

${ }^{\S}$ Statistical difference vs. Col group $(\mathrm{p}<0.05)$. 
was formed on the instrumented root following collagen hydrogel application to the furcation defect [27]. Therefore, collagen hydrogel is an essential factor for supplying periodontal ligament cells for periodontal regeneration. Furthermore, most of the implanted collagen scaffold in our experiment disappeared at 4 weeks, and inflammatory cells and root resorption were rarely observed; therefore, the scaffold has good biocompatibility. Collagen hydrogel is thus useful for facilitating proliferation of periodontal attachment cells.

Table 2. Frequency of aberrant periodontal healing events.

\begin{tabular}{|c|c|c|c|c|}
\hline & $\begin{array}{c}\text { Control } \\
\text { group }\end{array}$ & $\begin{array}{c}\text { BMP } \\
\text { group }\end{array}$ & $\begin{array}{c}\text { Col } \\
\text { group }\end{array}$ & $\begin{array}{c}\text { BMP/C } \\
\text { ol group }\end{array}$ \\
\hline \hline Ankylosis & $0 / 9$ & $6 / 9$ & $1 / 8$ & $1 / 8$ \\
\hline Epithelial downgrowth & $8 / 9$ & $5 / 9$ & $8 / 8$ & $5 / 8$ \\
\hline Gingival recession & $5 / 9$ & $1 / 9$ & $1 / 8$ & $0 / 8$ \\
\hline
\end{tabular}

We found that cementum-like tissue was significantly formed in the group receiving both BMP and hydrated collagen scaffold. It has been reported that implantation of BMP2 increased cementogenesis for periodontal attachment apparatus, as well as alveolar bone [16]. It is likely that cells derived from periodontal ligament attached to the BMPmodified root surface, and cementum formation was stimulated. We also speculate that BMP modification up-regulates the properties of periodontal ligament cells, such as synchronous regeneration of cementum and alveolar bone [27, 35] There were some differences in the structure of cementumlike tissue formed on the root surface between the BMP and hydrated collagen groups. Cementum-like tissue in the BMP group appeared to be thick and cellular, in contrast to that in the Col group. Miyaji et al. reported that cellular cementumlike tissue with no insertion of Sharpey's fibers, similar to a thin layer of bone tissue, was formed on BMP-applied dentin into or facing gingival connective tissue $[15,16]$. With regard to periodontal attachment and cementum-like tissue induced by BMP, it is necessary to clarify its long-term stability and functionality.

BMP release is an important issue in periodontal healing. In specimens receiving collagen hydrogel implants, BMP application promoted the restoration of alveolar bone loss, thus suggesting that the hydrated scaffold was gradually decorated with released BMP. We believe that collagen hydrogels can facilitate the capture and release of BMP. Hydrated materials possess water absorption properties similar to extracellular matrix; collagen hydrogel is able to retain water in the range of 20- to 200 -fold vs. dry weight $[22,25]$. A water-absorbable material is beneficial in the healing of tissue, as the regenerative space is able to retain supplementary tissue interstitial fluid containing several growth and nutritional factors that promote angiogenesis and tissue healing.

The other point to be considered is the relationship between periodontal healing and epithelial downgrowth. Periodontal regeneration is theoretically prevented by rapid epithelial cell downgrowth along the root surface in early healing stages. Therefore, clinical trials using barrier mem- brane (guided tissue regeneration therapy) have been performed after periodontal surgery [36, 37]. BMP application tended to suppress epithelial downgrowth in this study. Miyaji et al. also demonstrated that epithelial downgrowth is significantly prevented by root surface conditioning with BMP in dehiscence defects in dogs [16]. Some investigators demonstrated that BMP action on epidermal keratinocytes is similar to the effects of activin, which inhibits keratinocyte proliferation [38, 39]. Accordingly, BMP modification would be helpful for preventing the invasion of epithelium and promoting the proliferation of the periodontal cells. However, we used a no-infection model for periodontal examination; therefore, periodontal defects were likely to receive rich cells from healthy tissue, such as periodontal attachment and alveolar bone. In clinical settings, periodontal healing is confronted by dental plaque and inflammation. Further studies are thus necessary to elucidate the effects of infection on periodontal healing associated with BMP application.

\section{CONCLUSION}

We established a combination method using root surface modification with BMP and collagen hydrogel scaffold implantation to rebuild periodontal attachment. Single use of BMP produces severe ankylosis and limited reconstruction of the periodontal ligament. BMP application together with hydrated collagen scaffold provided new periodontal attachment including cementum-like tissue, periodontal ligament and alveolar bone. Our study also confirmed that BMPrelated ankylosis disappears with implantation of the collagen hydrogel. The combination method presented in this study will be useful for guiding predictable periodontal healing in advanced periodontitis.

\section{CONFLICT OF INTEREST}

The authors confirm that this article content has no conflict of interest.

\section{ACKNOWLEDGEMENTS}

We are grateful to Dr. Masaru Henmi, PhD, and our colleagues for their assistance. We would also like to thank Olympus Terumo Biomaterials Corp. for providing the FCHAC and Astellas Pharma Inc. for providing BMP-2. This work was supported by JPSP KAKENHI Grant Number 22791916 and 23792266.

\section{REFERENCES}

[1] Listgarten MA. Electron microscopic features of the newly formed epithelial attachment after gingival surgery. J Periodont Res 1967; 2: 46.

[2] Nyman S, Karring T, Lindhe J, Plantén S. Healing following implantation of periodontitis-affected roots into gingival connective tissue. J Clin Periodontol 1980; 7: 394-401.

[3] Lasho DJ, O'Leary TJ, Kafrawy AH. A scanning electron microscope study of the effects of various agents on instrumented periodontally involved root surfaces. J Periodontol 1983; 54: 210-20.

[4] Blomlöf J, Blomlöf L, Lindskog S. Effect of different concentrations of EDTA on smear removal and collagen exposure in periodontitis-affected root surfaces. J Clin Periodontol 1997; 24: 534-7.

[5] Pitaru S, Gray A, Aubin JE, Melcher AH. The influence of the morphological and chemical nature of dental surfaces on the migra- 
tion, attachment, and orientation of human gingival fibroblasts in vitro. J Periodont Res 1984; 19: 408-18.

[6] Fardal O, Lowenberg BF. A quantitative analysis of the migration, attachment, and orientation of human gingival fibroblasts to human dental root surfaces in vitro. J Periodontol 1990; 61: 529-35.

[7] Blomlöf J, Lindskog S. Root surface texture and early cell and tissue colonization after different etching modalities. Eur J Oral Sci 1995; 103: 17-24.

[8] Zaman KU, Sugaya T, Hongo O, Kato H. A study of attached and oriented human periodontal ligament cells to periodontally diseased cementum and dentin after demineralizing with neutral and low $\mathrm{pH}$ etching solution. J Periodontol 2000; 71: 1094-9.

[9] Katagiri T, Yamaguchi A, Ikeda T, et al. The non-osteogenic mouse pluripotent cell line, C3H10T 1/2, is induced to differentiate into osteoblastic cells by recombinant human bone morphogenetic protein-2. Biochem Biophys Res Commun 1990; 172: 295-9.

[10] Gong L, Hoshi K, Ejiri S, Nakajima T, Shingaki S, Ozawa H. Bisphosphonate incadronate inhibits maturation of ectopic bone induced by recombinant human bone induced by recombinant human bone morphogenetic protein-2. J Bone Miner Metab 2003; 21: 511.

[11] Kim SE, Jeon O, Lee JB, et al. Enhancement of ectopic bone formation by bone morphogenetic protein-2 delivery using heparinconjugated PLGA nanoparticles with transplantation of bone marrow-derived mesenchymal stem cells. J Biomed Sci 2008; 15: 7717.

[12] Zaman KU, Sugaya T, Kato H. Effect of recombinant human platelet-derived growth factor-BB and bone morphogenetic protein-2 application to demineralized dentin on early periodontal ligament cell response. J Periodont Res 1999; 34: 244-50.

[13] Miyaji H, Sugaya T, Miyamoto T, Kato K, Kato H. Hard tissue formation on dentin surfaces applied with recombinant human bone morphogenetic protein-2 in the connective tissue of the palate. $\mathrm{J}$ Periodont Res 2002; 37: 204-9.

[14] Miyaji H, Sugaya T, Kato K, Kawamura N, Tsuji H, Kawanami M. Dentin resorption and cementum-like tissue formation by bone morphogenetic protein application . J Periodont Res 2006; 41: 311-5.

[15] Miyaji H, Sugaya T, Ibe K, Ishizuka R, Tokunaga K, Kawanami M. Root surface conditioning with bone morphogenetic protein-2 facilitates cementum-like tissue deposition in beagle dogs. J Periodont Res 2010; 45: 658-63.

[16] Miyaji H, Sugaya T, Ibe K, Ishizuka R, Tokunaga K, Kawanami $M$. Influence of root dentin surface conditioning with bone morphogenetic protein-2 on periodontal wound healing in beagle dogs. J Oral Tissue Engin 2011; 8: 173-80.

[17] Saito E, Saito A, Kawanami M. Favorable healing following space creation in rhBMP-2-induced periodontal regeneration of horizontal circumferential defects in dogs with experimental periodontitis. J Periodontol 2003; 74: 1808-15.

[18] Saito Y, Yoshizawa T, Takizawa F, et al. A cell line with characteristics of the periodontal ligament fibroblasts is negatively regulated for mineralization and Runx2/Cbfa1/Osf2 activity, part of which can be overcome by bone morphogenetic protein-2. J Cell Sci 2002; 115: 4191-200.

[19] Akizuki T, Oda S, Komaki M, et al. Application of periodontal ligament cell sheet for periodontal regeneration: a pilot study in beagle dogs. J Periodont Res 2005; 40: 245-51.

[20] Inoue K, Miyaji H, Kosen Y, Sugaya T, Kawanami M. Ectopic bone induction by BMP-loaded collagen scaffold and bone marrow stromal cell sheet. J Oral Tissue Engin 2010; 8: 19-29.

[21] Chen FM, Jin Y. Periodontal tissue engineering and regeneration: current approaches and expanding opportunities. Tissue Engin B 2010; 16: 219-55.
[22] Park JB. The use of hydrogels in bone-tissue engineering. Med Oral Patol Oral Cir Bucal 2011; 16: 115-8.

[23] Hidaka T, Kinoshita Y, Fukuoka S, Ozono S, Kato K, Ikeda Y. A Study on the behaviors of periodontal ligament cells in a gel embedded collagen culture and their suitability for implant seeding. Jpn J Soc Biomater 1997; 15: 63-70.

[24] Matsui R, Ishikawa K, Takano Y, Katakura T. Application of collagen hydrogel material onto model delayed closing of fullthickness skin defect wound on guinea-pig. Jpn J Artif Organs 1997; $26: 772-8$

[25] Ishikawa K, Matsui R, Takano Y, Katakura T. Preparation of biodegradable hydrogel. Jpn J Artif Organs 1997; 26: 791-7.

[26] Miyaji H, Sugaya T, Kato K, Kawamura N, Kawanami M. The effects of collagen hydrogel implantation in buccal dehiscence defects in beagles. J Oral Tissue Engin 2007; 5: 87-95.

[27] Kosen Y, Miyaji H, Kato A, Sugaya T, Kawanami M. Application of collagen hydrogel/sponge scaffold facilitates periodontal wound healing in class II furcation defects in beagle dogs. J Periodont Res 2012; 47: 626-34.

[28] Kato A, Miyaji H, Kosen Y, et al. Periodontal healing by implantation of collagen hydrogel-sponge composite in one-wall infrabony defects in beagle dogs. J Oral Tissue Engin 2010; 8: 39-46.

[29] Sorensen RG, Wikesjö UM, Kinoshita A, Wozney JM. Periodontal repair in dogs: evaluation of a bioresorbable calcium phosphate cement (Ceredex) as a carrier for rhBMP-2. J Clin Periodontol 2004; 31: 796-804.

[30] Takahashi D, Odajima T, Morita M, Kawanami M, Kato H. Formation and resolution of ankylosis under application of recombinant human bone morphogenetic protein-2 (rhBMP-2) to class III furcation defects in cats. J Periodont Res 2005; 40: 299-305.

[31] Wikesjö UM, Guglielmoni P, Promsudthi A, et al. Periodontal repair in dogs: effect of rhBMP-2 concentration on regeneration of alveolar bone and periodontal attachment. J Clin Periodontal attachment. J Clin Periodontol 1999; 26: 392-400.

[32] Choi SH, Kim CK, Cho KS, et al. Effect of recombinant human bone morphogenetic protein-2/absorbable collagen sponge (rhBMP-2/ACS) on healing in 3-wall intrabony defects on dogs. J Periodontol 2002; 73: 63-72.

[33] Sigurdsson TJ, Lee MB, Kubota K, Turek TJ, Wozney JM, Wikesjö UM. Periodontal repair in dogs: recombinant human bone morphogenetic protein-2 significantly enhances periodontal regeneration. J Periodontol 1995; 66: 131-8.

[34] Lallier TE, Miner QW Jr, Sonnier J, Spencer A. A simple cell motility assay demonstrates differential motility of human periodontal ligament fibroblasts, gingival fibroblasts, and preosteoblasts. Cell Tissue Res 2007; 328: 339-54.

[35] Anzai A, Kitamura M, Nozaki T, et al. Effects of concomitant use of fibroblast growth factor (FGF)-2 with beta-tricalcium phosphate $(\beta-\mathrm{TCP})$ on the beagle dog 1-wall periodontal defect model. Biochem Biophys Res Commun 2010; 403: 345-50.

[36] Nyman S, Lindhe J, Karring T, Rylander H. New attachment following surgical treatment of human periodontal disease. J Clin Periodontol 1982; 9: 290-6.

[37] Gottlow J, Nyman S, Lindhe J, Karring T, Wennström J. New attachment formation in the human periodontium by guided tissue regeneration. J Clin Periodontol 1986; 13: 604-16.

[38] Seishima M, Nojiri M, Esaki C, Yoneda K, Eto Y, Kitajima Y. Activin A induces terminal differentiation of cultured human keratinocytes. J Invest Dermatol 1999; 112: 432-6.

[39] D'Souza SJ, Pajak A, Balazsi K, Dagnino L. Ca ${ }^{2+}$ and BMP-6 signaling regulate $\mathrm{E} 2 \mathrm{~F}$ during epidermal keratinocyte differentiation. $\mathrm{J}$ Biol Chem 2001; 276: 23531-8.

(C) Kato et al.; Licensee Bentham Open.

This is an open access article licensed under the terms of the Creative Commons Attribution Non-Commercial License (http://creativecommons.org/licenses/by-nc/3.0/) which permits unrestricted, non-commercial use, distribution and reproduction in any medium, provided the work is properly cited. 